\title{
Robust Phase-Based Features Extracted From Image By A Binarization Technique
}

\author{
Dr.T.Amitha, Dr.B.Raghu ${ }^{2}$ \\ ${ }^{1}$ Professor, Computer Science and Engineering, SVS Group of Institutions, Hanamkonda, Warangal -506 015, \\ ${ }^{2}$ Professor, Computer Science and Engineering, SVS Group of Institutions, Hanamkonda, Warangal -506 015,
}

\begin{abstract}
In this paper, a robust phase-based features extracted by binarization technique for ancient document images is proposed. Three feature maps derived from the phase information of an input document image constitute the core of this binarization model. These features are the maximum moment of phase congruency covariance, a locally weighted mean phase angle, and a phase preserved denoised image. The proposed model consists of three standard steps: 1) preprocessing; 2) main binarization; and 3) postprocessing. In the preprocessing and main binarization steps, the features used are mainly phase derived, while in the post processing step, specialized adaptive Gaussian and median filters are considered. One of the outputs of the binarization step, which shows high recall performance, is used in a proposed post processing method to improve the performance of other binarization methodologies
\end{abstract}

Keywords: component; formatting; style; styling; insert (key words)

\section{Introduction (Heading 1)}

Libraries, offices and archives contains an abundance of old and important documents. These documents having a significant amount of human heritage over time. However, many environmental factors and improper handling, of the materials used in their creation cause them to suffer a high degree of degradation of various types. Today, there is a strong move toward digitization of these manuscripts to preserve their content for future generations. The huge amount of digital data produced requires automatic processing, enhancement, and recognition. . The important step in all document image processing workflows is binarization, but this is not a very sophisticated process. Many studies have been carried out to solve the problems that arise in the binarization of old document images characterized by many types of degradation, including faded ink, bleedthrough, show-through, uneven illumination, variations in image contrast, and deterioration of the cellulose structure(Fig. 1). There are also differences in patterns of hand-written and machine-printed documents, which add to the difficulties associated with the binarization of old document images.

In this paper, a robust phase-based binarization method is proposed for the binarization and enhancement of historical documents and manuscripts. The three main steps in the proposed method are: preprocessing, main binarization, and post-processing. The preprocessing step mainly involves image denoising with phase preservation [24], followed by some morphological operations. We incorporate the Canny edge detector and a denoised image to obtain a binarized image in rough form.
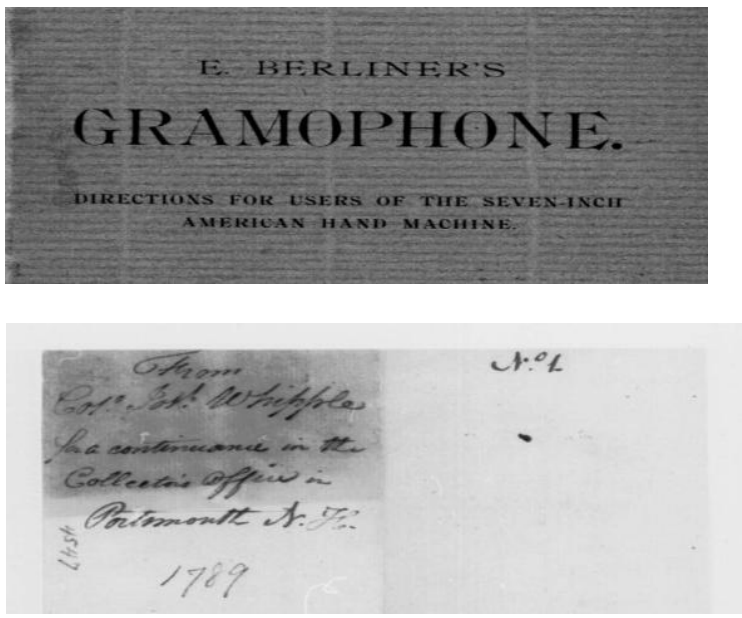

Fig 1

Then, we will use the phase congruency features for the main binarization step. Phase congruency is widely used in the machine vision and image processing literature palm print verification, object detection, 
finger-knuckle-print recognition, and biomedical applications. The phase congruency is a robust way to process historical documents, both handwritten and machine-printed manuscripts. After completing the three binarization steps on the input images using phase congruency features and a denoised image ,the enhancement processes are applied. A median filter and a phase congruency feature are used to construct an object exclusion map image.

The proposed binarization method is stable and robust to various types of degradation and to different datasets, thanks to its purpose-designed steps, and we provide comprehensive experimental results to demonstrate this robustness. The method outperforms most of the algorithms entered in the DIBCO'09 , H-DIBCO'10, DIBCO'11, H-DIBCO'12, DIBCO'13 and PHIBC'12 competitions, based on various evaluation measures, including the F-measure, NRM, PSNR, DRD, and MPM.

\section{Related Work}

In this section, we describe some selected binarization methods. Gatos et al. propose an adaptive binarization method based on low-pass filtering, foreground estimation, background surface computation, and a combination of these. An initial binary map is obtained using the multi-scale Sauvola's method and then statistical methods are used to restore the missed strokes and sub-strokes. Valizadeh et al. map input images into a two-dimensional feature space in which the foreground and background regions can be dis- tinguished. Then, they partition this feature space into several small regions, which are classified into text and background based on the results of applying Niblack's method.

Farrahi Moghaddam et al. propose a multi-scale bina

rization method in which the input document is binarized several times using different scales. Then, these output images are combined to form the final output image. This method uses different parameters for Sauvola's method to produce output images of the same size, but at different scales. In contrast, Lazzara and Gerard propose a multi-scale Sauvola's method which binarizes different scales of the input image with the same binarization parameters. Then, binary images with different scales are combined in some way to produce the final results.

Learning- based methods have also been proposed in recent years. These methods are an attempt to improve the outputs of other binarization methods based on a feature map or by determining the optimal parameters of binarization methods for each image . In and, a self-training document binarization method is proposed. The input pixels, depending on the binarization method(s) used, are divided into three categories: foreground, background, and uncertain, based on a prior knowledge about the behavior of every method used. Then, foreground and background pixels are clustered into different classes using the k-means algorithm or the random Markov field .Finally, uncertain pixels are classified with the label of their nearest neighboring cluster. The features used for the final decision are pixel intensity and local image contrast.

\section{Prhase -DERIVED FEATURES}

We use three phase-derived feature maps of the input document image in this paper: two phase congruency feature maps and a denoised image. The details are provided below.

\section{A. Phase Congruency-Based Feature Maps}

It is known that the phase information of an image outweighs its magnitude information. This implicitly means that phase information is the most important feature of images. In this section, two phase congruency-based feature maps extracted from input images are discussed. These feature maps are based on the Kovesi's phase congruency model. Another approach to the phase-based processing of images could be the monogenic scale-space method of . However, based on our experiments, Kovesi's method worked better within our proposed binarization method.

In phase congruency, the pixels of interest are at those points where the phase of the Fourier components is at its maximal. Let Me $\rho$ and Mo $\rho$ denote the even symmetric and odd symmetric log-Gabor wavelets at a scale $\rho$, which are known in the literature as quadratic pairs. By considering $f(x)$ as a onedimensional signal, the response of each quadratic pair of filters at each image point $\mathrm{x}$ forms a response vector by convolving with $\mathrm{f}(\mathrm{x})$ :

$$
\left[e_{\rho}(x), o_{\rho}(x)\right]=\left[f(x) * M_{\rho}^{e}, f(x) * M_{\rho}^{o}\right] \cdot \mid
$$

where values $e \rho(x)$ and $\rho \rho(x)$ are real and imaginary parts of a complex-valued wavelet response.

\section{B. Phase Preserving Denoising}

An image denoising method proposed by Kovesi is used in this paper, which is based on the assumption that phase information is the most important feature of images. This method also attempts to 
preserve the perceptually important phase information in the signal. It uses non-orthogonal, com- plex valued log-Gabor wavelets, which extract the local phase and amplitude information at each point in the image. The denoising process consists of determining a noise threshold at each scale and shrinking the magnitudes of the filter response vector appropriately, while leaving the phase unchanged. Auto- matic estimation of these noise thresholds, using the statistics of the smallest filter scale response, is the most important part of denoising. These statistics are used to estimate the distrib- ution of the noise amplitude, because they give the strongest noise response. Then, the noise amplitude distribution of other filter scales can be estimated proportionally.

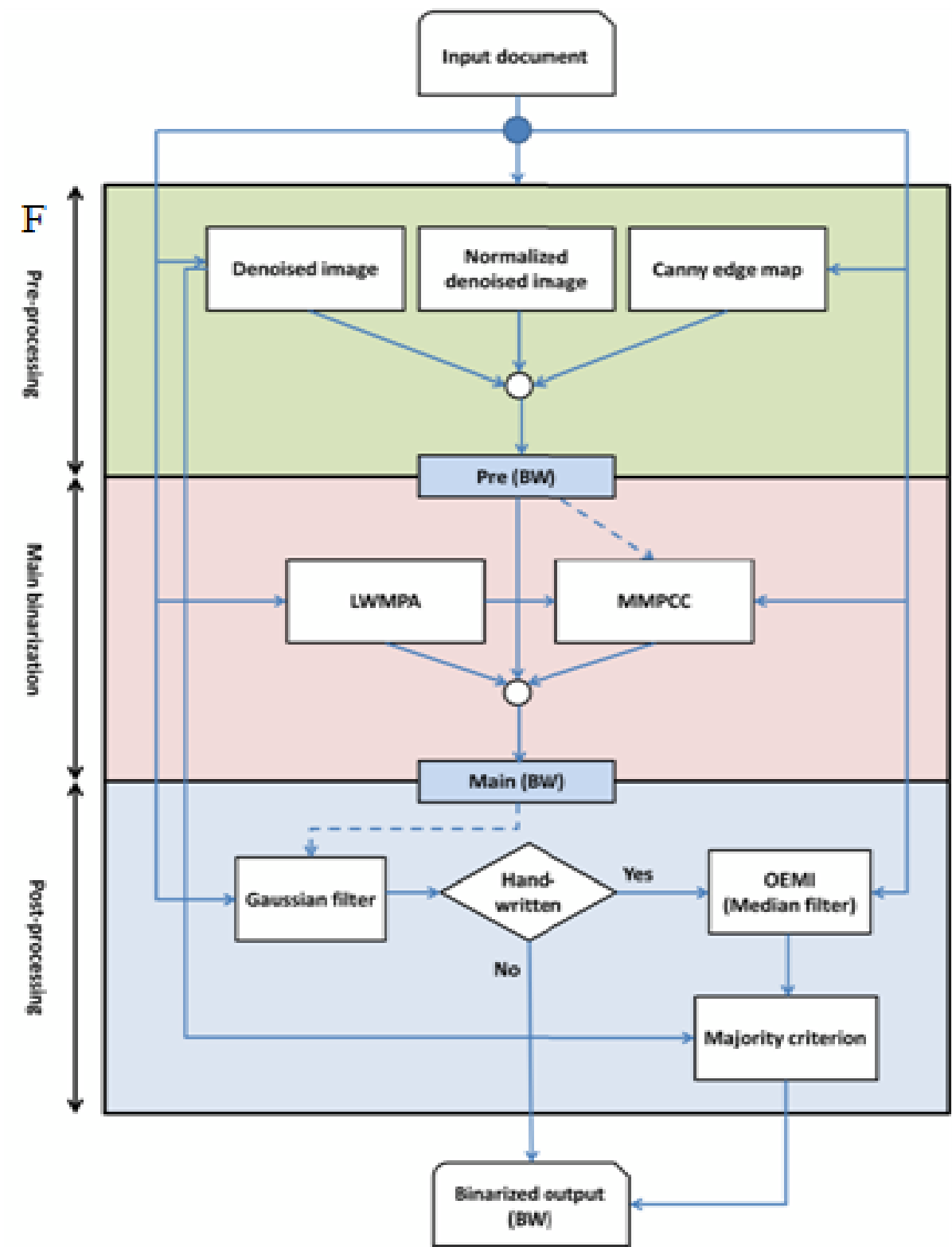

Fig. 2. Flowchart of the proposed binarization method. The solid lines show the binary output of this step, the dashed line shows that the binary output is also used to tune parameters of a step, and the white circles denote the pixel-wise AND operator.

\section{Binarization Model}

The final binarized output image is obtained by process- ing the input image in three steps: preprocessing, main binarization, and postprocessing. The binarization model is an extended version of the one proposed in our previous work. We have added a denoised image, which is another phase-based feature to the binarization model, and achieved 5\% improvement, on average. The flowchart of the proposed binarization method is shown in Fig. 2. Each step is discussed individually in the subsections below. 


\section{A. Preprocessing}

In the preprocessing step, we use a denoised image instead of the original image to obtain a binarized image in rough form. The image denoising method discussed in section III is applied to preprocess the binarization output. A number of parameters impact the quality of the denoised output image $\left(I_{D}\right)$, the key ones being the noise standard deviation threshold to be rejected $(k)$, and the number of filter scales $\left(N_{\rho}\right)$ and the number of orientations $\left(N_{r}\right)$ to be used. The $N_{\rho}$ parameter controls the extent to which low frequencies are covered. The higher $N_{\rho}$ is, the lower the frequencies, which means that the recall value remains optimal or near optimal.

We used Otsu's method on the normalized denoised image where normalized denoised image is obtained by applying a linear image transform on the denoised image. This approach can also remove noisy and degraded parts of images, because the denoising method attempts to shrink the amplitude infor- mation of the noise component. . See Fig. 3 for the output of Otsu's method when it is applied on a normalized denoised image.

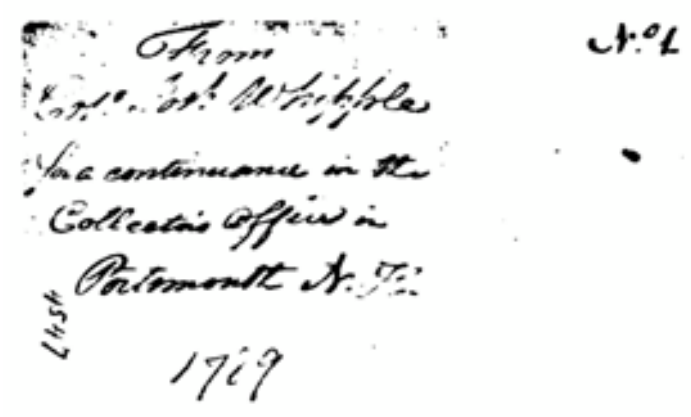

Fig 3. Output of Otsu's Method

\section{B. MAIN BINARIZATION}

The next step is the main binarization, which is based on phase congruency features: i) the maximum moment of phase congruency covariance $\left(I_{M}\right)$; and ii) the locally weighted mean phase angle $\left(I_{L}\right)$.

Fig. 4 shows an example of how we use $I_{M}$ to remove a majority of the background pixels.
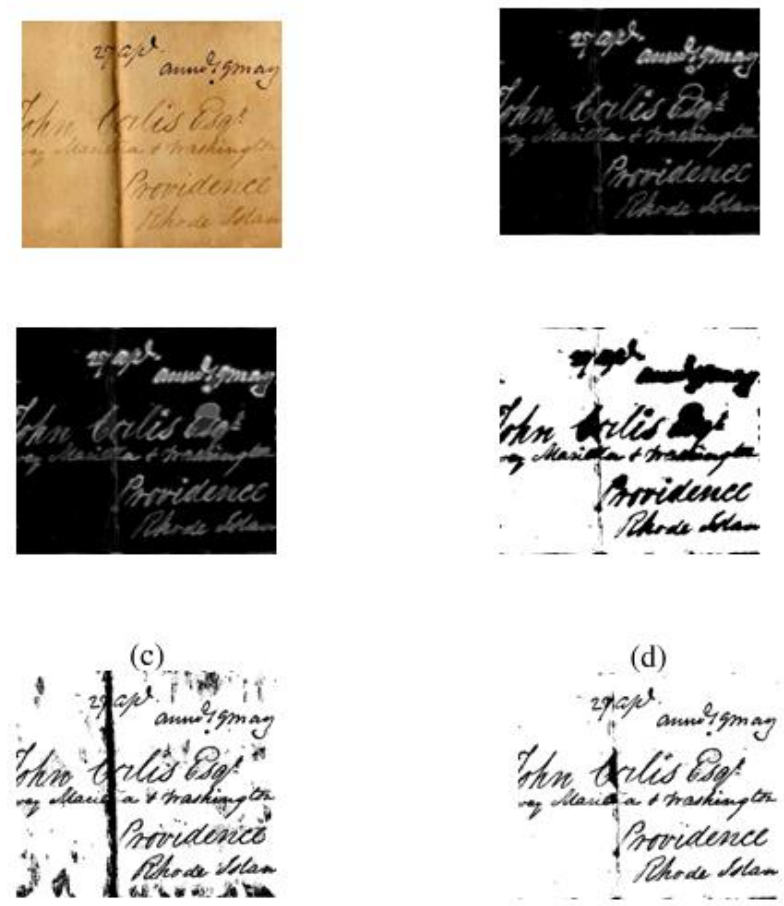

Fig. 4. A degraded document image and its binarized image using phase congruency. a) Original degraded document image. b) Edge image obtained by phase congruency $\left(I_{M}\right)$. c) Filled image of $I_{M}$. d) Binarization of (c) using Otsu's method. e) Denoised image and f) The result of main binarization. 


\section{Postprocessing}

In this step, we apply enhancement processes. First, a bleed- through removal process is applied. Then, a Gaussian filter is used to further enhance the binarization output and to separate background from foreground, and an exclusion process is applied, based on a median filter and IM maps, to remove background noise and objects. Finally, a further enhancement process is applied to the denoised image.

\section{References}

[1]. R. F. Moghaddam and M. Cheriet, "A multi-scale framework for adaptive binarization of degraded document images," Pattern Recognit., vol. 43, no. 6, pp. 2186-2198, 2010.

[2]. B. Su, S. Lu, and C. L. Tan, "Robust document image binarization technique for degraded document images," IEEE Trans, Image Process., vol. 22, no. 4, pp. 1408-1417, Apr. 2013.

[3]. K. Ntirogiannis, B. Gatos, and I. Pratikakis, "A combined approach for the binarization of handwritten document images," Pattern Recognit. Lett., vol. 35, pp. 3-15, Jan. 2014.

[4]. M. Valizadeh and E. Kabir, "Binarization of degraded document image based on feature space partitioning and classification," Int. J. Document Anal. Recognit., vol. 15, no. 1, pp. 57-69, 2010.

[5]. S. Lu, B. Su, and C. Tan, "Document image binarization using back- ground estimation and stroke edges," Int. J. Document Anal. Recognit., vol. 13, no. 4, pp. 303-314, 2010.

[6]. J. Sauvola and M. Pietikinen, "Adaptive

[7]. document image binarization,"

[8]. Pattern Recognit., vol. 33, no. 2, pp. 225-236, 2000

[9]. B. Gatos, I. Pratikakis, and S. Perantonis, “Adaptive degraded document image binarization,” Pattern Recognit., vol. 39, no. 3, pp. 317-327, 2006.

[10]. R. Hedjam, R. F. Moghaddam, and M. Cheriet, "A spatially adap- tive statistical method for the binarization of historical manuscripts and degraded document images," Pattern Recognit., vol. 44, no. 9, pp. 2184-2196, 2011. 